\title{
Determinant factors influencing people to use motorcycle taxi online services using the Analytical Hierarchy Process
}

\author{
Dr. Jozef Raco* (jraco@unikadelasalle.ac.id) \\ Mr. Yulius Raton (yraton@unikadelasalle.ac.id) \\ Mr. Frankie Taroreh (ftaroreh@unikadelasalle.ac.id) \\ Mr. Octavianus Muaja (omuaja@ unikadelasalle.ac.id) \\ Universitas Katolik De La Salle Manado - Indonesia \\ Kombos 1 Manado 95253 Indonesia
}

\section{*Corresponding author}

\begin{abstract}
The advancement of technology and Smartphone applications offers a lot of opportunities and challenges for companies to increase their market share. Through this technology and its application, companies such as transportation industries can make a lot of money and bring their products and services closer, faster and more easily to customers. In addition the customers can gain access to companies' services and products on time. On the other hand the advancement of Smartphone technology disrupts the common transportation business practices. Communication and negotiation are becoming more virtual. This technology brings about huge benefits to both customers and companies. However the same technology causes a huge problem especially to other transportation companies as they might lose market if they do not use it. This technology helps many transportation industries to make business innovations such as offering lower prices, faster services and deliveries. This research focuses on transportation companies, specifically motorcycle taxis with online booking, which use a Smartphone application.

In Manado Indonesia there are three popular motorcycle taxi online companies that use a Smartphone online application, which are Gojek, Grab and Uber. A lot of people use an online motorcycle taxi rather than public transportation because of its convenience, affordable price, safety and speed compared to local public transport. This study aims to find out the determinant factors that influence people to use motorcycle taxi online services. This research is going to reveal the favorite motorcycle taxi online company and its criteria based on respondents' perspectives. This paper will use the Analytical Hierarchy Process both for data gathering and data analysis. The research findings will contribute to the local government in formulating laws and policies specifically on motorcycle taxi online service.
\end{abstract}

Keywords: motorcycle taxi online service, analytical hierarchy process, transportation, Smartphone application.

Biographical Notes: Jozef Richard Raco is a lecturer in Business Management at the Universitas Katolik De La Salle Manado. He received his Ph.D in Educational Management from the State University of Jakarta-Indonesia in 2010. He earned his Master of Science in Business Management from the University of East Anglia UK in 2002, Master's in Economics 
from the Asian Social Institute of Manila-Philippines and Bachelor's in Philosophy from the Most Sacred Heart of Jesus Major Seminary of Pineleng, Manado Indonesia in 1990. His research interests are entrepreneurship, spirituality and values in Management.

Yulius Raton is a lecturer in modeling and simulation of the Industrial Engineering Department at the Universitas Katolik De La Salle Manado Indonesia. He earned his Master's in Computer science from School of Information Technology Benarif, Indonesia in 2008.

Franki Taroreh is a lecturer in Accounting, Finance and Management of the Accounting Department at the Universitas Katolik De La Salle Manado Indonesia. He got his Maters' in Accounting Management from the Universitas Sam Ratulangi Manado, Indonesia in 2009.

Octavianus Muaja is a lecturer in Accounting and Management of the Accounting Department at the Universitas Katolik De La Salle Manado Indonesia. He got his Masters' in Financial Management from the Universitas Sam Ratulangi Manado, Indonesia in 2011.

\section{Introduction}

Buying and selling products using the internet is quite common nowadays. The advancement of Information Technology and the use of a Smartphone application in business transaction become our common experiences. It makes it a lot of easier for people to do business. Business transactions are done faster and save time. Through this technology the geographical location is no longer a barrier. Rainer, Turban and Potter (Keong, 2015) mentioned that there are five added-values of Smartphone technology and its applications, namely: ubiquity, convenience, instant connectivity, personalization and localization of products and services. One of the most popular industries that use a Smartphone technology application is motorcycle taxi online services. The use of these online services is increasing. Sodikin (2017) added that by December 2017 the number of people in the country using motorcycle taxi online services reached 15 million per week. Furthermore he wrote that by 2018 this number is expected to rise due to the change in people's behavior moving from conventional transaction, to online service systems which is through a Smartphone application. Conventional transaction or offline system has gradually been replaced by the online service (Liu, 2014). It is also happening in Manado city in Indonesia (Fig.1). 
Figure 1. Map of Manado city - Indonesia

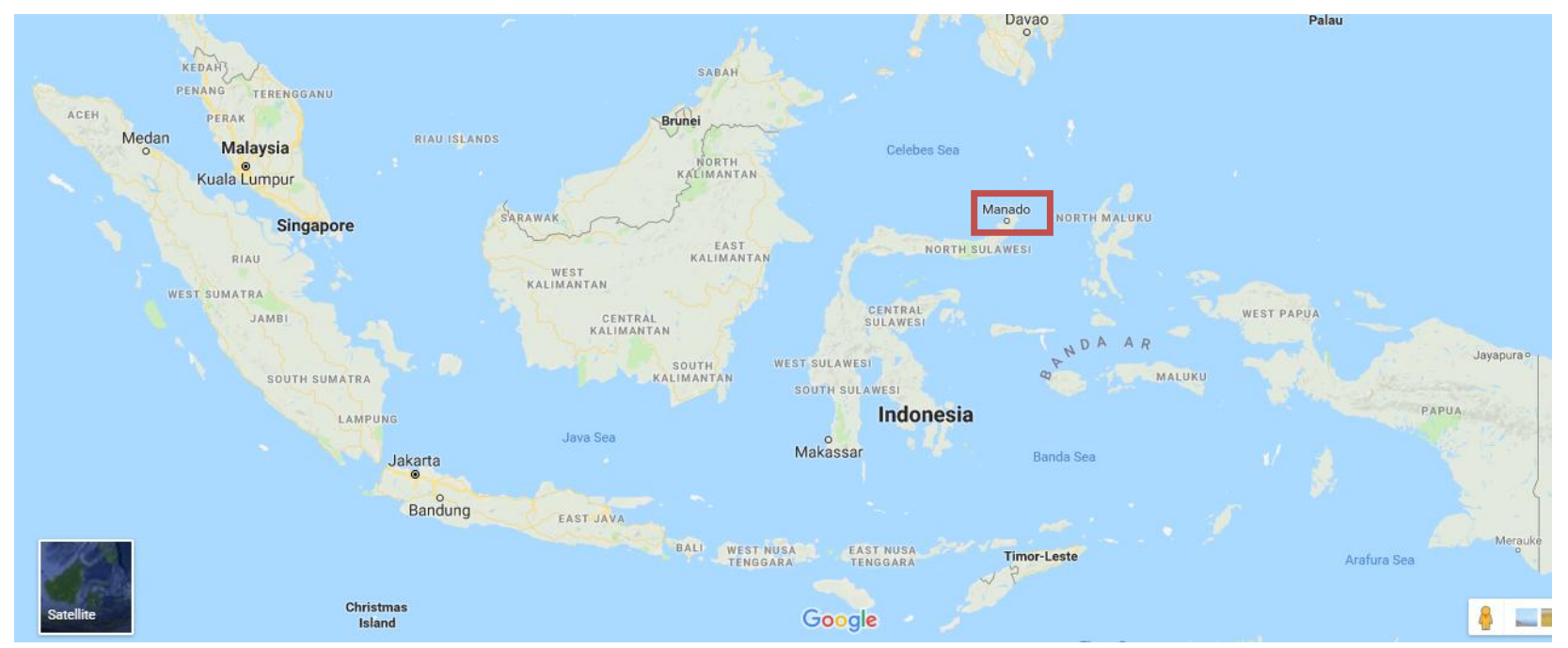

Source : google map

People in Manado are getting more familiar with online motorcycle taxi services than the offline one since it is more convenient, faster, cheaper and easier to access.

This paper is aiming to find out the dominant factors which influence people in Manado to use online motorcycle taxi. There are three motorcycle taxi online companies that operate in Manado that are namely Gojek, Grabike and Uberbike.

Figure 2: Grab bike

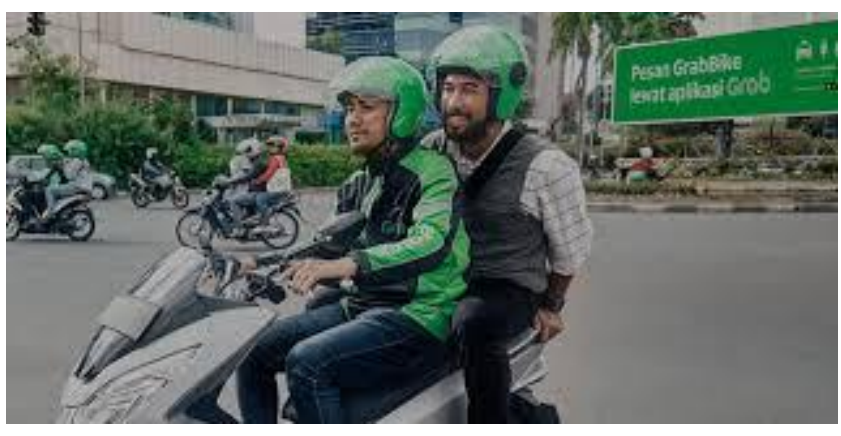

Source: google.co.id 
Figure 3: Gojek

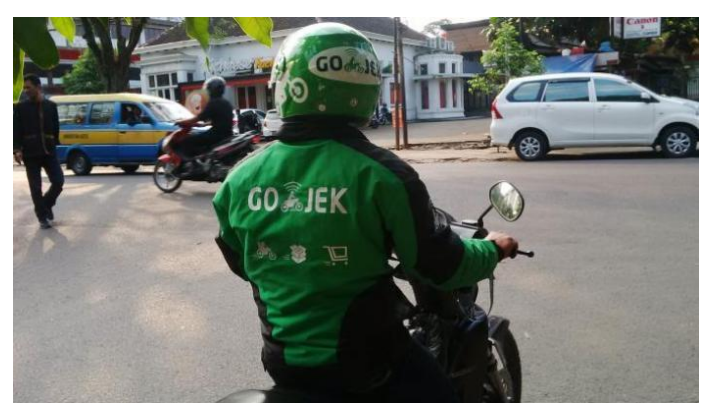

Source: google.co.id

Figure 4: Uberbike

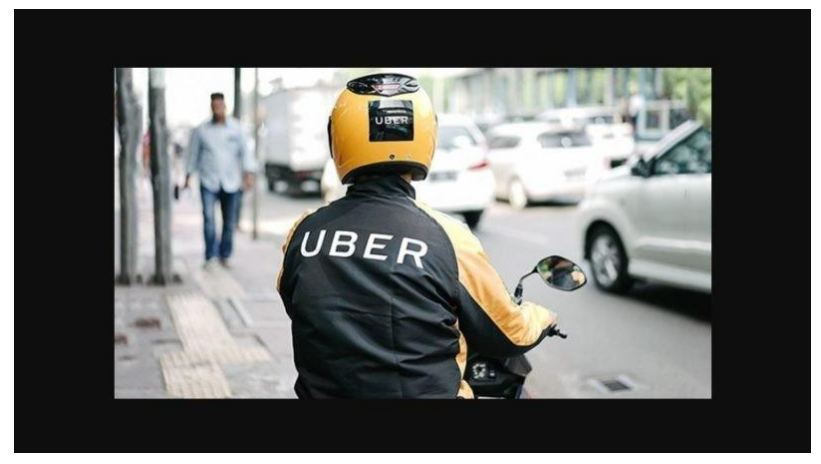

Source:google.co.id

\section{Literature review}

The theory of Technology Acceptance Model (TAM) is commonly used to explain the behavior of people who are using IT based services such as Smartphone applications. This theory mentioned that if we want to determine the success of an online service system we have to see how people perceive its usefulness, ease of use and their attitude toward the usage of the system (Shah, et al, 2013). They added that perceived usefulness is understood as the degree to which a person believes that using a particular system would enhance his or her performance. Perceived ease of use indicates the degree to which a person believes that using a particular system would be free of effort. While attitude towards usage represents the degree to which an individual evaluates and associates the target system with his or her job. Many studies reveal that the Technological Acceptance Model has been widely used by information technology researchers to gain a better understanding of information technology (IT) adoption and its usage in organizations (Liu. 2014). 
That theory works on motorcycle taxi online services because, as mentioned by Karema, (2013) motorcycle taxis can provide speed travel, easy maneuverability, ability to pass on poor and small size road (Kumar, 2011). Moreover a motorcycle taxi offers convenience trip and affordable prices for people's mobility because it allows the rider to weave through congestion especially during rush hours. It is suitable to those demanding flexible and door to door mobility (Qian, 2015). Through the Smartphone application, mobility becomes a much cheaper, direct, relatively quick and personal service with a reliable travel time. The motorcycle taxi is optimizing usage of road space by sharing a limited public space that a maximum of people can use it.

Another theory which supports this study is the theory of perceived benefits, perceived privacy and trust (Zhang.G. et al, 2017). Zhang explained that perceived benefits are important to understand the customers' behavior. He added that perceived benefits in online services are quite numerous mainly because of their convenience, ease of use, system quality, attitude, value creation, users' satisfaction, trust and commitment. Furthermore he stated that when users perceived benefits, they will generate positive attitude and emotion that will strengthen their trust in operators.

Secondly, perceived privacy referred to the perceived risk due to users disclosing their personal information on the systems. All data about the customers are guaranteed safe. The perceived privacy is considered as one of the important aspects of perceived risk. Some researches revealed that when users perceived risk, they would feel worried and anxious and this would lead to their decline in trust toward the operator. In this context as customers using motorcycle taxi online services disclose their personal information they will perceive high privacy risk. So the more privacy risk they perceive, the more worried and anxious they feel and the more reluctant they are to trust motorcycle taxi online services.

Trust is defined as the users' belief that the operator is behaving ethically. The more trust users perceive, the more positive behavior they will generate and the more likely they are to promote and recommend the company to their friends.

Through the literature review the researchers identified some factors considered as reasons for people to use motorcycle taxi online services. These factors were classified as criteria and sub criteria for this research. These criteria are: convenience, price, safety, and speed. The sub criteria of convenience are: pick and drop on site, easy to access, personal, predictable time. The 
sub-criteria of price are: affordable price, fixed (nonnegotiable price), flexible payment (cash or credit). The sub-criteria of safety are: traceable route, identified driver, complete document of the vehicle, acknowledged headquarter. The sub-criteria of speed are: real time service, access all roads, direct trip, and maneuverability.

\section{Methodology}

This study uses the Analytical Hierarchy Process (AHP) for data gathering and analysis. This method was introduced by Thomas Saaty in the 1970s and commonly used to the research related to a decision making process where many and complex variables and factors are involved. This method is also a combination of qualitative and quantitative technique. SinuanyStern et.al (2006) wrote that there are reasons to use the AHP method. First it is applicable to many disciplines. Second it is widely and ease to use. This method allows researchers to take into account all important criteria and to organize them into a hierarchy. This method uses consistency validation where the researcher can eliminate redundant data and an algorithm checks to see if your input is consistent. Sinuany-Stern et al (2006) added that the AHP has become more popular because it provides an opportunity for a richer involvement of the decision makers in the evaluating procedure. It is much more widely taught in management workshop and textbooks. Banuelas and Anthony (2004) mentioned that the AHP is used by many researchers because it helps them understand the context of the problem and make it easy for them to structure the problem to be solved. It assists the researcher in managing the relationship between people and their deferential willingness and ability to adjust to the changed circumstances of the desired state of the problem. It helps the researchers reach a consensus, generate new insights and have more confidence in the results. It provides them with more than one method to tackle real-world problems.

The Analytical Hierarchy Process starts with the establishment of the hierarchical structure. The hierarchy structure displays the criteria, sub criteria and alternatives. It then weights the elements of different levels. And the calculation of the weight of the elements on different levels is completed through the following steps:

a. Formation of a hierarchical structure. Through the hierarchy structure the problems were broken down.

b. Establishment of pair-wise comparison matrix 
c. Calculation of the priority vector

d. Calculation of the maximum Eigen value

e. Examination of the consistency

Data collection was done through a questionnaire with AHP format. The questionnaire was distributed to 50 respondents that were selected purposely. There are some criteria for selecting respondents. First the respondents were regular users of motorcycle taxi online services. In other words the respondents have direct experiences of the service (Raco \& Tanod, 2014). They were also willing to participate in this study and allow the researcher to publish the data.

\section{Results}

Through the literature and previous studies, the researchers determined 4 factors considered as the criteria for this study. These criteria are convenience, price, safety and speed. Each criterion has sub-criteria. The sub-criteria of convenience are: pick and drop on the spot, easy to access, privatization, predictable time to arrive. The sub-criteria of price are affordability, flexibility payment, fixedness price. The sub-criteria of safety are traceability route; driver can be recognized, having a formal office, having valid license and good condition of the vehicle. The online hailing motorcycle companies (Gojek, Grabike and Uberbike) operating in Manado Indonesia, were the three alternatives used in this research. The criteria, the sub-criteria and the alternatives were organized in hierarchical form as shown in Fig.5 
Figure 5: The hierarchical structure of the study

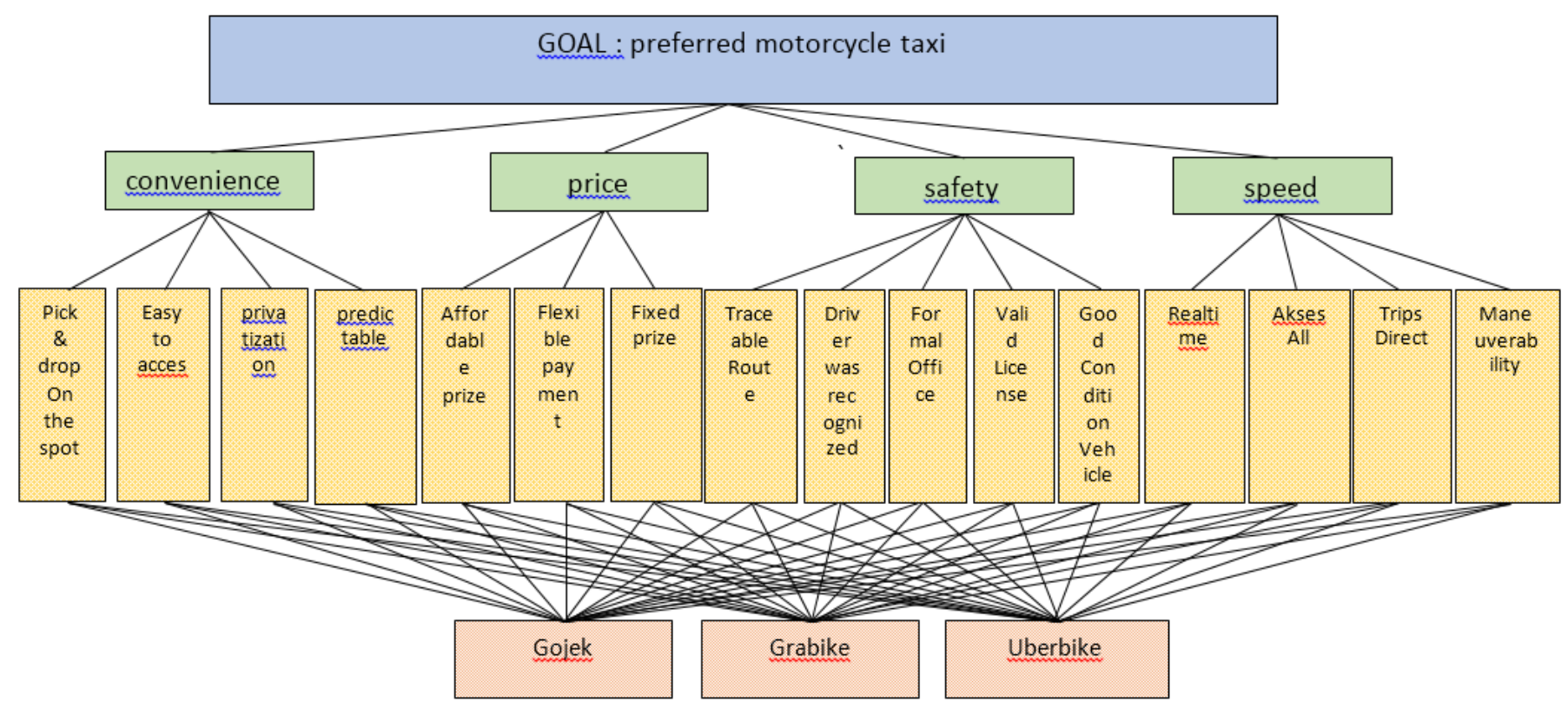

Subsequently the AHP questionnaire was transformed into a pairwise comparisons form based on the hierarchy structure of the criteria, sub-criteria and the alternatives. Table 1 below shows a typical nine-point scale for an AHP questionnaire introduced by Saaty.

Table 1. Saaty's 9 point scale / The definition and explanation of the AHP 9 point scale

\begin{tabular}{|c|c|}
\hline $\begin{array}{c}\text { Intensity of relative } \\
\text { importance }\end{array}$ & Definition \\
\hline 1 & Equal Importance \\
\hline 3 & Moderate importance of one over another \\
\hline 5 & Essential or strong importance \\
\hline 7 & Demonstrated importance \\
\hline 9 & Absolute importance \\
\hline $2,4,6,8$ & Intermediate values between the two neighboring \\
& scales \\
\hline
\end{tabular}

The respondents were asked to tick the desired answers from a scale of 1 to 9, using Saaty scale, for a total of eighteen questions.

The questionnaires, as appeared in table 2 and table 3, were distributed to 50 respondents who have previous experience of the service and the information for this study (Raco \& Tanod, 
2014). Only 15 respondents returned the questionnaire and the researchers considered that number as sufficient for analysis. For this research the qualification of the respondents are of bigger importance than their number, as they have the important information needed by researchers, having first hand experience as using the motorcycle taxi online services and as they are willing to participate in this research and allow the researchers to publish the findings.

Table 2. Weighting criteria by paired comparison using questionnaire format by respondent 1 .

\begin{tabular}{|c|c|c|c|c|c|c|c|c|c|c|c|c|c|c|c|c|c|c|}
\hline \multirow[t]{3}{*}{ Criteria } & \multicolumn{17}{|c|}{ Criteria Weighting Score } & \multirow[t]{3}{*}{ Criteria } \\
\hline & \multicolumn{8}{|c|}{ More importance than } & Equal & \multicolumn{8}{|c|}{ Less importance than } & \\
\hline & 9 & 8 & 7 & 6 & 5 & 4 & 3 & 2 & 1 & 2 & 3 & 4 & 5 & 6 & 7 & 8 & 9 & \\
\hline Convinience & 9 & 8 & 7 & 6 & 5 & 4 & (3) & 2 & 1 & 2 & 3 & 4 & 5 & 6 & 7 & 8 & 9 & Price \\
\hline Convinience & 9 & 8 & 7 & 6 & 5 & 4 & (3) & 2 & 1 & 2 & 3 & 4 & 5 & 6 & 7 & 8 & 9 & Safety \\
\hline Convinience & 9 & 8 & 7 & 6 & 5 & 4 & 3 & 2 & 1 & 2 & 3 & 4 & 5 & 6 & 7 & 8 & 9 & Speed \\
\hline Price & 9 & 8 & (7) & 6 & 5 & 4 & 3 & 2 & 1 & 2 & 3 & 4 & 5 & 6 & 7 & 8 & 9 & Safety \\
\hline Price & (9) & 8 & 7 & 6 & 5 & 4 & 3 & 2 & 1 & 2 & 3 & 4 & 5 & 6 & 7 & 8 & 9 & Speed \\
\hline Safety & 9 & 8 & 7 & 6 & 5 & 4 & 3 & 2 & (1) & 2 & 3 & 4 & 5 & 6 & 7 & 8 & 9 & Speed \\
\hline
\end{tabular}

Table 3. Weighting sub-criteria with respect to the convenience by paired comparison using questionnaire format by respondent 1

\begin{tabular}{|c|c|c|c|c|c|c|c|c|c|c|c|c|c|c|c|c|c|c|}
\hline \multirow[t]{3}{*}{ SubCriteria } & \multicolumn{17}{|c|}{ Sub-Criteria Weighting Score } & \multirow[t]{3}{*}{ Subcriteria } \\
\hline & \multicolumn{8}{|c|}{ More importance than } & Equal & \multicolumn{8}{|c|}{ Less importance than } & \\
\hline & 9 & 8 & 7 & 6 & 5 & 4 & 3 & 2 & 1 & 2 & 3 & 4 & 5 & 6 & 7 & 8 & 9 & \\
\hline Pick \& Drop & 9 & 8 & 7 & 6 & 5 & 4 & 3 & 2 & (1) & 2 & 3 & 4 & 5 & 6 & 7 & 8 & 9 & Easy to Acces \\
\hline Pick \& Drop & 9 & 8 & 7 & 6 & 5 & 4 & 3 & 2 & (1) & 2 & 3 & 4 & 5 & 6 & 7 & 8 & 9 & Privatization \\
\hline Pick \& Drop & 9 & 8 & 7 & 6 & 5 & 4 & 3 & 2 & 1 & 2 & 3 & 4 & 5 & 6 & 7 & 8 & 9 & Predictable \\
\hline Easy to Acces & 9 & 8 & (7) & 6 & 5 & 4 & 3 & 2 & 1 & 2 & 3 & 4 & 5 & 6 & 7 & 8 & 9 & Privatization \\
\hline Easy to Acces & 9 & 8 & 7 & 6 & (5) & 4 & 3 & 2 & 1 & 2 & 3 & 4 & 5 & 6 & 7 & 8 & 9 & Predictable \\
\hline Privatization & 9 & 8 & 7 & 6 & 5 & 4 & 3 & 2 & (1) & 2 & 3 & 4 & 5 & 6 & 7 & 8 & 9 & Predictable \\
\hline
\end{tabular}

Once the data was collected, the researchers used geometric mean calculation to get the mean and to eliminate deviation. The formula of the geometric mean, formula 1 , ca be described as follows:

$$
G M=\sqrt[n]{\left(x_{1}\right)\left(x_{2}\right) \ldots\left(x_{n}\right)}
$$

with :

GM: Geometric Mean

$x_{1}$ : weighting by first respondent

$x_{n}:$ weighting by $n^{\text {th }}$ respondent

$n:$ number of respondents

The result of geometric mean calculation are showen on the table 4 and table 5 below : 
Table 4 . The results of weighting criteria by 15 respondents and calculation the associated geometric mean $(\mathrm{GM})$ in paired comparison

\begin{tabular}{|l|c|c|c|c|c|c|c|c|c|c|c|c|c|c|c|c|l|l|}
\hline & \multicolumn{10}{|c|}{ Respondent } & \multicolumn{1}{|c|}{ GM } \\
Criteria & $\mathbf{1}$ & $\mathbf{2}$ & $\mathbf{3}$ & $\mathbf{4}$ & $\mathbf{5}$ & $\mathbf{6}$ & $\mathbf{7}$ & $\mathbf{8}$ & $\mathbf{9}$ & $\mathbf{1 0}$ & $\mathbf{1 1}$ & $\mathbf{1 2}$ & $\mathbf{1 3}$ & $\mathbf{1 4}$ & $\mathbf{1 5}$ & Criteria & \\
\hline Convenience & 1 & 1 & 1 & 1 & 1 & 1 & 1 & 1 & 1 & 3 & 1 & 3 & 3 & 1 & 3 & Price & 1.340394 \\
\hline Convenience & 3 & 1 & 2 & 3 & 3 & 1 & 3 & 3 & 3 & 1 & 3 & 1 & 1 & 1 & 1 & Safety & 1.748740 \\
\hline Convenient & 3 & 1 & 3 & 3 & 1 & 3 & 3 & 1 & 2 & 5 & 1 & 3 & 1 & 1 & 3 & Speed & 1.946809 \\
\hline Price & 7 & 5 & 7 & 7 & 7 & 5 & 5 & 5 & 3 & 5 & 1 & 1 & 5 & 3 & 3 & Safety & 3.984481 \\
\hline Price & 9 & 7 & 6 & 7 & 7 & 7 & 5 & 3 & 3 & 1 & 1 & 3 & 3 & 5 & 3 & Speed & 3.918220 \\
\hline Safety & 1 & 3 & 2 & 1 & 1 & 3 & 3 & 3 & 2 & 1 & 1 & 1 & 4 & 3 & 1 & Speed & 1.735062 \\
\hline
\end{tabular}

Table 5. The results of weighting sub criteria with respect to convenience by 15 respondents and calculation of the associated geometric mean (GM) in paired comparison.

\begin{tabular}{|c|c|c|c|c|c|c|c|c|c|c|c|c|c|c|c|c|c|}
\hline \multirow[b]{2}{*}{ Subcriteria } & \multicolumn{15}{|c|}{ Respondent } & \multirow[b]{2}{*}{ Subcriteria } & \multirow[b]{2}{*}{ GM } \\
\hline & 1 & 2 & 3 & 4 & 5 & 6 & 7 & 8 & 9 & 10 & 11 & 12 & 13 & 14 & 15 & & \\
\hline Pick \& Drop & 1 & 1 & 1 & 1 & 1 & 1 & 1 & 3 & 3 & 1 & 1 & 3 & 1 & 3 & 1 & Easy to Access & 1.340394 \\
\hline Pick \& Drop & 1 & 3 & 1 & 3 & 1 & 3 & 3 & 4 & 3 & 3 & 3 & 2 & 2 & 3 & 3 & Privatization & 2.325666 \\
\hline Pick \& Drop & 1 & 5 & 1 & 3 & 1 & 3 & 5 & 7 & 5 & 2 & 4 & 1 & 2 & 7 & 5 & Predictable & 2.773081 \\
\hline Easy to Access & 7 & 7 & 5 & 5 & 5 & 4 & 5 & 7 & 7 & 4 & 3 & 2 & 3 & 1 & 7 & Privatization & 4.285987 \\
\hline Easy to Access & 5 & 5 & 3 & 5 & 7 & 3 & 5 & 5 & 7 & 5 & 3 & 5 & 3 & 5 & 7 & Predictable & 4.666980 \\
\hline Privatization & 1 & 3 & 3 & 3 & 1 & 1 & 1 & 2 & 1 & 7 & 3 & 3 & 1 & 3 & 3 & Predictable & 1.990972 \\
\hline
\end{tabular}

Once the geometric mean was calculated, the researchers established the pairwise comparison matrix. All the criteria do not bear the same importance. Therefore, the next step in the AHP process is to derive the relative priority weights for the criteria, sub-criteria and alternatives. To perform the pair-wise comparison, using the formula (2 and 3), we need to create a comparison matrix of the criteria, subcriteria and alternative involved in the decision from the results before, using the formula below:

$$
A=\left(a_{i j}\right)_{n \times n}
$$

Saaty added that each element of the matrix is representing ratio and weight of the criteria 


$$
A=\left(w_{i} / w_{j}\right)_{n x n}=\left[\begin{array}{cccc}
w_{1} / w_{1} & w_{1} / w_{2} & \ldots & w_{1} / w_{n} \\
w_{2} / w_{1} & w_{2} / w_{2} & \ldots & w_{2} / w_{n} \\
\vdots & \vdots & \vdots & \vdots \\
w_{n} / w_{1} & w_{n} / w_{2} & \ldots & w_{n} / w_{n}
\end{array}\right]
$$

with : $a_{i j}=\frac{1}{a_{j i}}$, for $i \neq j$, and $a_{i i}=1$, all $i$

The pairwise comparison matrix for the criteria is showen in table 6, while table 7 presents the pairwise comparison matrix for the sub criteria.

Tabel 6. Pairwise comparison matrix of criteria with respect to the goal

\begin{tabular}{|l|c|l|l|l|}
\hline & Convenience & Price & Safety & Speed \\
\hline Convenience & 1 & 1.340393566 & 1.748740032 & 1.946808583 \\
\hline Price & 0.746049537 & 1 & 3.984480723 & 3.918219967 \\
\hline Safety & 0.571840286 & 0.250973733 & 1 & 1.735062341 \\
\hline Speed & 0.513661183 & 0.255217933 & 0.576348167 & 1 \\
\hline
\end{tabular}

Tabel 7. Pairwise comparison matrix of sub criteria.

\begin{tabular}{|c|c|c|c|c|c|}
\hline CONVENIENCE & Pick \& Drop & Easy to Access & Privatization & Predictable & \\
\hline Pick \& Drop & 1 & 1.340393566 & 2.325666399 & 2.773081111 & \\
\hline Easy to Access & 0.746049537 & 1 & 4.285986976 & 4.666980055 & \\
\hline Privatization & 0.429984283 & 0.233318488 & 1 & 1.990971857 & \\
\hline Predictability & 0.360609719 & 0.214271325 & 0.50226727 & 1 & \\
\hline PRICE & Affordable & Fixed & Flexible & & \\
\hline Affordable & 1 & 3.395261313 & 4.71769398 & & \\
\hline Fixed & 0.29452814 & 1 & 2.377160938 & & \\
\hline Flexible & 0.211967967 & 0.420669877 & 1 & & \\
\hline SAFETY & Traceable Route & Driver Recognized & Formal Office & Valid License & G.C. Vehicle \\
\hline Traceable Route & 1 & 2.232099863 & 2.448222887 & 2.634262426 & 2.105674 \\
\hline Driver Recognized & 0.448008629 & 1 & 2.634262426 & 2.172572128 & 2.14800863 \\
\hline Formal Office & 0.408459542 & 0.3796129 & 1 & 1.885191985 & 1.60845954 \\
\hline Valid License & 0.3796129 & 0.460283913 & 0.530449953 & 1 & 1.3796129 \\
\hline G.C. Vehicle & 0.474907322 & 0.465547478 & 0.621712871 & 0.724841004 & 1 \\
\hline SPEED & Realtime & All Acces & Direct Trips & Maneuverability & \\
\hline Realtime & 1 & 1.800059738 & 2.854418322 & 1.761066482 & \\
\hline All Access & 0.555537118 & 1 & 1.998494699 & 1.648231337 & \\
\hline Direct Trips & 0.350334074 & 0.500376609 & 1 & 1.44105564 & \\
\hline Maneuverablity & 0.567837734 & 0.60671095 & 0.693935732 & 1 & \\
\hline
\end{tabular}


Table 8 and table 9 below present the pairwise comparison matrix for alternatives.

Tabel 8. Pairwise comparison matrix of alternatives (part I)

\begin{tabular}{|c|c|c|c|}
\hline \multicolumn{4}{|c|}{ CONVENIENCE } \\
\hline Pick \& Drop & Go-ride & Grab-bike & Uber - bike \\
\hline Go-ride & 1 & 0.428826137 & 2.96719735 \\
\hline Grab-bike & 2.331947413 & 1 & 3.325733936 \\
\hline Uber-bike & 0.337018365 & 0.300685509 & 1 \\
\hline Easy to Acces & Go-ride & Grab-bike & Uber - bike \\
\hline Go-ride & 1 & 0.398647063 & 4.016908774 \\
\hline Grab-bike & 2.508484553 & 1 & 4.648119937 \\
\hline Uber-bike & 0.24894765 & 0.215140748 & 1 \\
\hline Privatization & Go-ride & Grab-bike & Uber - bike \\
\hline Go-ride & 1 & 0.428826137 & 3.191825287 \\
\hline Grab-bike & 2.331947413 & 1 & 3.091682043 \\
\hline Uber-bike & 0.313300356 & 0.323448526 & 1 \\
\hline Predictable & Go-ride & Grab-bike & Uber - bike \\
\hline Go-ride & 1 & 0.985061205 & 3 \\
\hline Grab-bike & 1.015165347 & 1 & 2.5642542 \\
\hline Uber-bike & 0.333333333 & 0.389976938 & 1 \\
\hline \multicolumn{4}{|c|}{ PRICE } \\
\hline Affordable & Go-ride & Grab-bike & Uber - bike \\
\hline Go-ride & 1 & 0.291768854 & 1.152453457 \\
\hline Grab-bike & 3.427370629 & 1 & 1.909587062 \\
\hline Uber-bike & 0.867714001 & 0.523673427 & 1 \\
\hline Fixed & Go-ride & Grab-bike & Uber - bike \\
\hline Go-ride & 1 & 0.398647063 & 2.191799867 \\
\hline Grab-bike & 2.508484553 & 1 & 2.934753372 \\
\hline Uber-bike & 0.456246036 & 0.340744135 & 1 \\
\hline Flexible & Go-ride & Grab-bike & Uber - bike \\
\hline Go-ride & 1 & 0.8547514 & 3.191825287 \\
\hline Grab-bike & 1.169930813 & 1 & 3.191825287 \\
\hline Uber-bike & 0.313300356 & 0.313300356 & 1 \\
\hline \multicolumn{4}{|c|}{ SAFETY } \\
\hline Traceable Route & Go-ride & Grab-bike & Uber - bike \\
\hline Go-ride & 1 & 0.40423228 & 2.191799867 \\
\hline Grab-bike & 2.473825195 & 1 & 2.473825195 \\
\hline Uber-bike & 0.456246036 & 0.40423228 & 1 \\
\hline
\end{tabular}


Tabel 9. Pairwise comparison matrix of alternatives (part II)

\begin{tabular}{|c|c|c|c|}
\hline \multicolumn{4}{|c|}{ SAFETY } \\
\hline Driver Recognized & Go-ride & Grab-bike & Uber - bike \\
\hline Go-ride & 1 & 0.580532829 & 2.728217132 \\
\hline Grab-bike & 1.722555471 & 1 & 2.728217132 \\
\hline Uber-bike & 0.366539741 & 0.366539741 & 1 \\
\hline Formal Office & Go-ride & Grab-bike & Uber - bike \\
\hline Go-ride & 1 & 1.087595747 & 2.728217132 \\
\hline Grab-bike & 0.919459278 & 1 & 2.728217132 \\
\hline Uber-bike & 0.366539741 & 0.366539741 & 1 \\
\hline Valid License & Go-ride & Grab-bike & Uber - bike \\
\hline Go-ride & 1 & 0.679183244 & 2.728217132 \\
\hline Grab-bike & 1.4723567 & 1 & 2.728217132 \\
\hline Uber-bike & 0.366539741 & 0.366539741 & 1 \\
\hline G.C. Vehicle & Go-ride & Grab-bike & Uber - bike \\
\hline Go-ride & 1 & 0.366539741 & 2.191799867 \\
\hline Grab-bike & 2.728217132 & 1 & 2.728217132 \\
\hline Uber-bike & 0.456246036 & 0.366539741 & 1 \\
\hline \multicolumn{4}{|c|}{ SPEED } \\
\hline Realtime & Go-ride & Grab-bike & Uber - bike \\
\hline Go-ride & 1 & 0.8547514 & 3.433458398 \\
\hline Grab-bike & 1.169930813 & 1 & 3.433458398 \\
\hline Uber-bike & 0.291251527 & 0.291251527 & 1 \\
\hline All Access & Go-ride & Grab-bike & Uber - bike \\
\hline Go-ride & 1 & 0.794597405 & 3.156925178 \\
\hline Grab-bike & 1.258498951 & 1 & 3.156925178 \\
\hline Uber-bike & 0.316763922 & 0.316763922 & 1 \\
\hline Direct Trips & Go-ride & Grab-bike & Uber - bike \\
\hline Go-ride & 1 & 0.679183244 & 2.728217132 \\
\hline Grab-bike & 1.4723567 & 1 & 2.728217132 \\
\hline Uber-bike & 0.366539741 & 0.366539741 & 1 \\
\hline Manueverability & Go-ride & Grab-bike & Uber - bike \\
\hline Go-ride & 1 & 0.231427707 & 2.934753372 \\
\hline Grab-bike & 4.321003791 & 1 & 6.512527832 \\
\hline Uber-bike & 0.340744135 & 0.153550208 & \\
\hline
\end{tabular}


The next step consist in obtaining the normalized pairwise comparison matrix (PCM), as written in formula 4 and 5, and generating the priority vector (formula 6) awarding to the following :

a. Sum each column in the pairwise comparison matrix

$$
a_{i j}=\sum_{i=1}^{n} a_{i j}
$$

b. Create the normalized pairwise matrix .

$$
X_{i, j}=\frac{a_{i j}}{\sum_{i=1}^{n} a_{i j}}
$$

c. The priroty vector by dividing the sum of the normalized column matrix by the number of criteria/sub criteria/alternatives.

$$
W_{i, j}=\frac{\sum_{j=1}^{n} X_{i j}}{n}
$$

The results are shown in the table $10,11,12,13$ and 14 below :

Tabel 10. Normalized PCM and Priority Vector of Criteria with respect to the goal

\begin{tabular}{|l|c|l|l|l|c|}
\hline & Convenience & Price & Safe & Speed & Priority Vector \\
\hline Convenience & 0.353163336 & 0.470877721 & 0.239239831 & 0.226370699 & 0.322413 \\
\hline Price & 0.263477344 & 0.351298106 & 0.545104748 & 0.455602158 & 0.403871 \\
\hline Safety & 0.201953023 & 0.088166597 & 0.136806973 & 0.201749303 & 0.157169 \\
\hline Speed & 0.181406297 & 0.089657576 & 0.078848448 & 0.116277841 & 0.116548 \\
\hline
\end{tabular}

Tabel 11. Normalized PCM and Priority Vector of Sub Criteria (part I)

\begin{tabular}{|l|c|c|c|c|c|c|}
\hline CONVENIENCE & Pick \& Drop & $\begin{array}{l}\text { Easy to } \\
\text { Access }\end{array}$ & Privatization & Predictable & & $\begin{array}{l}\text { Priority } \\
\text { Vector }\end{array}$ \\
\hline Pick \& Drop & 0.394221728 & 0.480775307 & 0.286626712 & 0.265849135 & & 0.356868 \\
\hline Easy to Access & 0.294108938 & 0.358682196 & 0.528226386 & 0.447413027 & & 0.407108 \\
\hline Privatization & 0.169509147 & 0.083687188 & 0.123244982 & 0.190870056 & & 0.141828 \\
\hline Predictable & 0.142160187 & 0.076855309 & 0.06190192 & 0.095867782 & & 0.094196 \\
\hline PRICE & Affordable & Fixed & Flexile & & & $\begin{array}{l}\text { Priority } \\
\text { Vector }\end{array}$ \\
\hline Affordable & 0.663791958 & 0.705006193 & 0.582801548 & & & 0.650533 \\
\hline Fixed & 0.195505411 & 0.207644163 & 0.293663192 & & & 0.232271 \\
\hline Flexible & 0.140702632 & 0.087349644 & 0.123535259 & & & 0.117196 \\
\hline SAFETY & Traceable Route & $\begin{array}{l}\text { Rriver } \\
\text { Recognized }\end{array}$ & Formal Office & Valid License & & \\
\hline Traceable Route & 0.368869156 & 0.491918048 & 0.338402482 & 0.312974205 & 0.255488544 & 0.353530487 \\
\hline Driver Recognized & 0.165256565 & 0.22038353 & 0.36411756 & 0.258121221 & 0.260625147 & 0.253700805 \\
\hline Formal Office & 0.150668127 & 0.083660431 & 0.138223723 & 0.223977861 & 0.195159833 & 0.158337995 \\
\hline Valid License & 0.14002749 & 0.101438994 & 0.073320767 & 0.118809046 & 0.167393096 & 0.120197879 \\
\hline G.C. Vehicle & 0.175178663 & 0.102598997 & 0.085935468 & 0.086117668 & 0.12133338 & 0.114232835 \\
\hline
\end{tabular}


Tabel 12. Normalized PCM and Priority Vector of Sub Criteria (part II)

\begin{tabular}{|l|c|c|c|c|c|l|}
\hline SPEED & Realtime & All Access & Direct Trips & Maneuverability & & $\begin{array}{l}\text { Priority } \\
\text { Vector }\end{array}$ \\
\hline Realtime & 0.40425128 & 0.460709464 & 0.435998819 & 0.301018818 & & 0.400495 \\
\hline All Access & 0.224576591 & 0.255941208 & 0.305260557 & 0.281731924 & & 0.266878 \\
\hline Direct Trips & 0.141622998 & 0.128066994 & 0.152745242 & 0.246319415 & & 0.167189 \\
\hline Maneuverablity & 0.229549131 & 0.155282334 & 0.105995382 & 0.170929843 & & 0.165439 \\
\hline
\end{tabular}

Tabel 13. Normalized PCM and Priority Vector of Alternatives (part I)

\begin{tabular}{|l|l|l|l|c|}
\hline \multicolumn{5}{|c|}{ CONVENIENCE } \\
\hline Pick \& Drop & Go-ride & Grab-bike & Uber - bike & Priority Vector \\
\hline Go-ride & 0.272556372 & 0.247946371 & 0.406859359 & 0.309120701 \\
\hline Grab-bike & 0.635587126 & 0.578197899 & 0.456021565 & 0.556602196 \\
\hline Uber-bike & 0.091856503 & 0.173855729 & 0.137119076 & 0.134277103 \\
\hline Easy to Access & Go-ride & Grab-bike & Uber - bike & Priority Vector \\
\hline Go-ride & 0.2661392 & 0.2470257 & 0.41561271 & 0.309592536 \\
\hline Grab-bike & 0.667606072 & 0.619660152 & 0.480921483 & 0.589395902 \\
\hline Uber-bike & 0.066254728 & 0.133314149 & 0.103465807 & 0.101011561 \\
\hline Privatization & Go-ride & Grab-bike & Uber - bike & Priority Vector \\
\hline Go-ride & 0.274329775 & 0.244725411 & 0.438226412 & 0.319093866 \\
\hline Grab-bike & 0.639722609 & 0.570686789 & 0.424477096 & 0.544962165 \\
\hline Uber-bike & 0.085947616 & 0.184587801 & 0.137296491 & 0.135943969 \\
\hline Predictable & Go-ride & Grab-bike & Uber - bike & Priority Vector \\
\hline Go-ride & 0.425803944 & 0.414755952 & 0.457020692 & 0.432526863 \\
\hline Grab-bike & 0.432261408 & 0.421045869 & 0.390639077 & 0.414648785 \\
\hline Uber-bike & 0.141934648 & 0.164198179 & 0.152340231 & 0.152824353 \\
\hline \multicolumn{5}{|c|}{ PRICE } \\
\hline Affordable & Go-ride & Grab-bike & Uber - bike & Priority Vector \\
\hline Go-ride & 0.188854394 & 0.160715026 & 0.28371294 & 0.21109412 \\
\hline Grab-bike & 0.647274004 & 0.550829961 & 0.47010537 & 0.556069778 \\
\hline Uber-bike & 0.163871602 & 0.288455013 & 0.24618169 & 0.232836102 \\
\hline Fixed & Go-ride & Grab-bike & Uber - bike & Priority Vector \\
\hline Go-ride & 0.252223948 & 0.229187697 & 0.357754153 & 0.279721933 \\
\hline Grab-bike & 0.632699876 & 0.574913798 & 0.479021932 & 0.562211869 \\
\hline Uber-bike & 0.115076176 & 0.195898505 & 0.163223914 & 0.158066198 \\
\hline Flexible & Go-ride & Grab-bike & Uber - bike & Priority Vector \\
\hline Go-ride & 0.402701131 & 0.394248614 & 0.432282819 & 0.409744188 \\
\hline Grab-bike & 0.471132461 & 0.461243601 & 0.432282819 & 0.454886294 \\
\hline Uber-bike & 0.126166408 & 0.144507785 & 0.135434361 & 0.135369518 \\
\hline
\end{tabular}


Tabel 14. Normalized PCM and Priority Vector of Alternatives (part II)

\begin{tabular}{|c|c|c|c|c|}
\hline \multicolumn{5}{|c|}{ SAFETY } \\
\hline Traceable Route & Go-ride & Grab-bike & Uber - bike & Priority Vector \\
\hline Go-ride & 0.254448314 & 0.223522368 & 0.386859321 & 0.288276668 \\
\hline Grab-bike & 0.629460651 & 0.552955265 & 0.436637647 & 0.539684521 \\
\hline Uber-bike & 0.116091035 & 0.223522368 & 0.176503032 & 0.172038811 \\
\hline Driver Recognized & Go-ride & Grab-bike & Uber - bike & Priority Vector \\
\hline Go-ride & 0.323719384 & 0.29815675 & 0.422557873 & 0.348144669 \\
\hline Grab-bike & 0.557624597 & 0.51359154 & 0.422557873 & 0.49792467 \\
\hline Uber-bike & 0.118656019 & 0.18825171 & 0.154884253 & 0.153930661 \\
\hline Formal Office & Go-ride & Grab-bike & Uber - bike & Priority Vector \\
\hline Go-ride & 0.437445507 & 0.443168583 & 0.422557873 & 0.434390655 \\
\hline Grab-bike & 0.40221333 & 0.407475465 & 0.422557873 & 0.41074889 \\
\hline Uber-bike & 0.160341163 & 0.149355951 & 0.154884253 & 0.154860456 \\
\hline Valid License & Go-ride & Grab-bike & Uber - bike & Priority Vector \\
\hline Go-ride & 0.352249552 & 0.332001571 & 0.422557873 & 0.368936332 \\
\hline Grab-bike & 0.518636988 & 0.488824737 & 0.422557873 & 0.4766732 \\
\hline Uber-bike & 0.12911346 & 0.179173692 & 0.154884253 & 0.154390468 \\
\hline G.C. Vehicle & Go-ride & Grab-bike & Uber - bike & Priority Vector \\
\hline Go-ride & 0.238979281 & 0.211496209 & 0.370235401 & 0.273570297 \\
\hline Grab-bike & 0.651987369 & 0.577007581 & 0.460846165 & 0.563280372 \\
\hline Uber-bike & 0.10903335 & 0.211496209 & 0.168918434 & 0.163149331 \\
\hline \multicolumn{5}{|c|}{ SPEED } \\
\hline Realtime & Go-ride & Grab-bike & Uber - bike & Priority Vector \\
\hline Go-ride & 0.406308783 & 0.39829927 & 0.436442699 & 0.413683584 \\
\hline Grab-bike & 0.475353164 & 0.465982589 & 0.436442699 & 0.459259484 \\
\hline Uber-bike & 0.118338053 & 0.13571814 & 0.127114602 & 0.127056932 \\
\hline All Access & Go-ride & Grab-bike & Uber - bike & Priority Vector \\
\hline Go-ride & 0.388309873 & 0.376343639 & 0.431636556 & 0.398763356 \\
\hline Grab-bike & 0.488687568 & 0.473628075 & 0.431636556 & 0.464650733 \\
\hline Uber-bike & 0.123002558 & 0.150028286 & 0.136726888 & 0.136585911 \\
\hline Direct Trips & Go-ride & Grab-bike & Uber - bike & Priority Vector \\
\hline Go-ride & 0.352249552 & 0.332001571 & 0.422557873 & 0.368936332 \\
\hline Grab-bike & 0.518636988 & 0.488824737 & 0.422557873 & 0.4766732 \\
\hline Uber-bike & 0.12911346 & 0.179173692 & 0.154884253 & 0.154390468 \\
\hline Manueverability & Go-ride & Grab-bike & Uber - bike & Priority Vector \\
\hline Go-ride & 0.1766239 & 0.167098482 & 0.280910728 & 0.208211037 \\
\hline Grab-bike & 0.763192542 & 0.722033174 & 0.623370588 & 0.702865435 \\
\hline Uber-bike & 0.060183558 & 0.110868344 & 0.095718683 & 0.088923528 \\
\hline
\end{tabular}


Based on the calculation, as shown in table 10, the researchers found that the criteria which has the highest value was price $(40 \%)$ followed by convenience $(32 \%)$, safety $16 \%$ and speed (12 $\%)$.

\section{Consistency}

The purpose of a consistency test is to ensure whether the calculation fits the condition of transitivity in priority. A consistency ratio $(\mathrm{CR})$ is used to verify the credibility and reasonability of the evaluation and to check whether there is inconsistent causality or conflicts in subjective judgments. The CR is acceptable if it does not exceed 0.1 or less than $10 \%$. The definition of the consistency index is shown in the formula (7).

Since the numeric values are derived from the subjective preferences of individuals, it is impossible to avoid some inconsistencies in the final matrix of judgments. The question is how much inconsistency is acceptable. For this purpose, AHP calculates a consistency ratio (CR) comparing the consistency index (CI) of the matrix in question (the one with our judgments) versus the consistency index of a random-like matrix (see formula 8). A random matrix is one where the judgments have been entered randomly and therefore it is expected to be highly inconsistent. Saaty provides the calculated RI value for matrices of different sizes as shown in Table 15. In AHP, the consistency ratio is defined as CR where CR = CI/RI (formula 8). Saaty has shown that a consistency ratio (CR) of 0.10 or less than $10 \%$ is acceptable to continue the AHP analysis. If the consistency ratio is greater than 0.10 , it is necessary to revise the judgments to locate the cause of the inconsistency and correct it.

The value of Random Index for different numbers of criteria is shown in the table 15 below.

Table 15. Random index value

\begin{tabular}{|c|c|c|c|c|c|c|c|c|c|c|c|c|c|c|c|}
\hline $\mathrm{N}$ & 1 & 2 & 3 & 4 & 5 & 6 & 7 & 8 & 9 & 10 & 11 & 12 & 13 & 14 & 15 \\
\hline R.I & 0.00 & 0.00 & 0.58 & 0.90 & 1.12 & 1.24 & 1.32 & 1.41 & 1.45 & 1.49 & 1.51 & 1.48 & 1.56 & 1.57 & 1.59 \\
\hline
\end{tabular}

The principle eigen value $(\lambda \max )$ is obtained as follows :

- Multiply each element of the priority vector with the sum of each corresponding column in the pairwise matrix .

- The principal eigen value is obtained by summing the results from the first step above. 
The consistency Index is obtained as follows : $C I=\frac{\lambda_{\max }-n}{(n-1)}$

The last step is the calculation of the Consistency Ratio as follows $C R=\frac{C I}{R I}$.

RI values refer to table 15 above.

Table 16 below is used to calculate the consistency

Tabel 16. PCM and Priority Vector of the Criteria

\begin{tabular}{|l|c|l|l|l|c|}
\hline & Convenience & Price & Safe & Speed & Priority Vector \\
\hline Convenient & 1 & 1.340393566 & 1.748740032 & 1.946808583 & 0.322413 \\
\hline Price & 0.746049537 & 1 & 3.984480723 & 3.918219967 & 0.403871 \\
\hline Safety & 0.571840286 & 0.250973733 & 1 & 1.735062341 & 0.157169 \\
\hline Speed & 0.513661183 & 0.255217933 & 0.576348167 & 1 & 0.116548 \\
\hline SUM & 2.831551007 & 2.846585232 & 7.309568921 & & \\
\hline
\end{tabular}

The consistency calculation for the criteria unfolds as follows

$$
\begin{aligned}
& \begin{array}{r}
(\lambda \max )=(0,322413 * 2.831551007)+(0.403871 * 2.846585232)+(0.157169 * 7.309568921) \\
+(0.116548 * 8.600090891)=4.213738
\end{array} \\
& C I=\frac{\lambda_{\max }-n}{(n-1)}=\frac{4.213738-4}{3}=0.071246 \\
& C R=\frac{C I}{R I}=\frac{0,071246}{0,90}=0.079162
\end{aligned}
$$

The result of $\lambda \max , \mathrm{CI}$ and CR for sub criteria and alternatives appear in table 17, 18, 19 and 20 below:

Table 17. Values of $\lambda \max , \mathrm{CI}$ and $\mathrm{CR}$ for Criteria

\begin{tabular}{|l|c|c|c|c|}
\hline CRITERIA & Priority Vector & $\lambda \max$ & CI & CR \\
\cline { 1 - 2 } Convenience & 0.322413 & \multirow{2}{*}{4.213738} & \multirow{2}{*}{0.071246} & 0.079162 \\
\cline { 1 - 2 } Price & 0.403871 & & & \\
\cline { 1 - 2 } Safety & 0.157169 & & & \\
\cline { 1 - 2 } Speed & 0.116548 & & & \\
\hline
\end{tabular}


Table 18. Values of $\lambda \max , \mathrm{CI}$ and $\mathrm{CR}$ for Sub Criteria

\begin{tabular}{|c|c|c|c|c|}
\hline CONVENIENCE & Priority Vector & $\lambda \max$ & CI & $\mathbf{C R}$ \\
\hline Pick \& Drop & 0.356868 & \multirow{4}{*}{4.173601} & \multirow{4}{*}{0.0578671} & \multirow{4}{*}{0.0642968} \\
\hline Easy to Access & 0.407108 & & & \\
\hline Privatization & 0.141828 & & & \\
\hline Predictable & 0.094196 & & & \\
\hline PRICE & Priority Vector & $\lambda \max$ & CI & CR \\
\hline Affordable & 0.650533 & \multirow{3}{*}{3.04731} & \multirow{3}{*}{0.023655} & \multirow{3}{*}{0.040784} \\
\hline Fixed & 0.232271 & & & \\
\hline Flexible & 0.117196 & & & \\
\hline SAFETY & Priority Vector & $\lambda \max$ & CI & $\mathrm{CR}$ \\
\hline Traceable Route & 0.353530487 & \multirow{5}{*}{5.208284} & \multirow{5}{*}{0.052071} & \multirow{5}{*}{0.046492} \\
\hline Driver Recognized & 0.253700805 & & & \\
\hline Formal Office & 0.158337995 & & & \\
\hline Valid License & 0.120197879 & & & \\
\hline G.C. Vehicle & 0.114232835 & & & \\
\hline SPEED & Priority Vector & $\lambda \max$ & CI & CR \\
\hline Realtime & 0.400495 & \multirow{4}{*}{4.095874} & \multirow{4}{*}{0.031958} & \multirow{4}{*}{0.035509} \\
\hline All Access & 0.266878 & & & \\
\hline Direct Trips & 0.167189 & & & \\
\hline Maneuverablity & 0.165439 & & & \\
\hline
\end{tabular}


Table 19. Values of $\lambda \max , \mathrm{CI}$ and CR for Alterntive (part I)

\begin{tabular}{|c|c|c|c|c|}
\hline \multicolumn{5}{|c|}{ CONVENIENCE } \\
\hline Pick \& Drop & Priority Vector & $\lambda \max$ & CI & $\mathbf{C R}$ \\
\hline Go-ride & 0.309120701 & \multirow{3}{*}{3.076077} & \multirow{3}{*}{0.038038} & \multirow{3}{*}{0.065584} \\
\hline Grab-bike & 0.556602196 & & & \\
\hline Uber-bike & 0.134277103 & & & \\
\hline Easy to Access & Priority Vector & $\lambda \max$ & CI & CR \\
\hline Go-ride & 0.309592536 & \multirow{3}{*}{3.090713} & \multirow{3}{*}{0.045356} & \multirow{3}{*}{0.0782} \\
\hline Grab-bike & 0.589395902 & & & \\
\hline Uber-bike & 0.101011561 & & & \\
\hline Privatization & Priority Vector & $\lambda \max$ & CI & $\mathbf{C R}$ \\
\hline Go-ride & 0.319093866 & \multirow{3}{*}{3.108248} & \multirow{3}{*}{0.054124} & \multirow{3}{*}{0.093318} \\
\hline Grab-bike & 0.544962165 & & & \\
\hline Uber-bike & 0.135943969 & & & \\
\hline Predictable & Priority Vector & $\lambda \max$ & CI & CR \\
\hline Go-ride & 0.432526863 & \multirow{3}{*}{3.003773} & \multirow{3}{*}{0.001887} & \multirow{3}{*}{0.003253} \\
\hline Grab-bike & 0.414648785 & & & \\
\hline Uber-bike & 0.152824353 & & & \\
\hline \multicolumn{5}{|c|}{ PRICE } \\
\hline Affordable & Priority Vector & $\lambda \max$ & CI & $\mathbf{C R}$ \\
\hline Go-ride & 0.21109412 & \multirow{3}{*}{3.073063} & \multirow{3}{*}{0.036532} & \multirow{3}{*}{0.062986} \\
\hline Grab-bike & 0.556069778 & & & \\
\hline Uber-bike & 0.232836102 & & & \\
\hline Fixed & Priority Vector & $\lambda \max$ & CI & $\mathbf{C R}$ \\
\hline Go-ride & 0.279721933 & \multirow{3}{*}{3.055329} & \multirow{3}{*}{0.027665} & \multirow{3}{*}{0.047698} \\
\hline Grab-bike & 0.562211869 & & & \\
\hline Uber-bike & 0.158066198 & & & \\
\hline Flexible & Priority Vector & $\lambda \max$ & CI & $\mathbf{C R}$ \\
\hline Go-ride & 0.409744188 & \multirow{3}{*}{3.003228} & \multirow{3}{*}{0.001614} & \multirow{3}{*}{0.002783} \\
\hline Grab-bike & 0.454886294 & & & \\
\hline Uber-bike & 0.135369518 & & & \\
\hline
\end{tabular}


Table 20. Values of $\lambda \max , \mathrm{CI}$ and CR for Alterntive (part II)

\begin{tabular}{|c|c|c|c|c|}
\hline \multicolumn{5}{|c|}{ SAFETY } \\
\hline Traceable Route & Priority Vector & $\lambda \max$ & CI & $\mathbf{C R}$ \\
\hline Go-ride & 0.288276668 & \multirow{3}{*}{3.083656} & \multirow{3}{*}{0.041828} & \multirow{3}{*}{0.072117} \\
\hline Grab-bike & 0.539684521 & & & \\
\hline Uber-bike & 0.172038811 & & & \\
\hline Driver Recognized & Priority Vector & $\lambda \max$ & CI & CR \\
\hline Go-ride & 0.348144669 & \multirow{3}{*}{3.038791} & \multirow{3}{*}{0.019395} & \multirow{3}{*}{0.03344} \\
\hline Grab-bike & 0.49792467 & & & \\
\hline Uber-bike & 0.153930661 & & & \\
\hline Formal Office & Priority Vector & $\lambda \max$ & CI & $\mathbf{C R}$ \\
\hline Go-ride & 0.434390655 & \multirow{3}{*}{3.000896} & \multirow{3}{*}{0.000448} & \multirow{3}{*}{0.000773} \\
\hline Grab-bike & 0.41074889 & & & \\
\hline Uber-bike & 0.154860456 & & & \\
\hline Valid License & \begin{tabular}{|l|} 
Priority Vector \\
\end{tabular} & $\lambda \max$ & CI & CR \\
\hline Go-ride & 0.368936332 & \multirow{3}{*}{3.019325} & \multirow{3}{*}{0.009663} & \multirow{3}{*}{0.01666} \\
\hline Grab-bike & 0.4766732 & & & \\
\hline Uber-bike & 0.154390468 & & & \\
\hline G.C. Vehicle & Priority Vector & $\lambda \max$ & CI & $\mathbf{C R}$ \\
\hline Go-ride & 0.273570297 & \multirow{3}{*}{3.086801} & \multirow{3}{*}{0.043401} & \multirow{3}{*}{0.074829} \\
\hline Grab-bike & 0.563280372 & & & \\
\hline Uber-bike & 0.163149331 & & & \\
\hline \multicolumn{5}{|c|}{ SPEED } \\
\hline Realtime & Priority Vector & $\lambda \max$ & CI & $\mathbf{C R}$ \\
\hline Go-ride & 0.413683584 & \multirow{3}{*}{3.003269} & \multirow{3}{*}{0.001635} & \multirow{3}{*}{0.002818} \\
\hline Grab-bike & 0.459259484 & & & \\
\hline Uber-bike & 0.127056932 & & & \\
\hline All Access & Priority Vector & $\lambda \max$ & CI & $\mathbf{C R}$ \\
\hline Go-ride & 0.398763356 & \multirow{3}{*}{3.006935} & \multirow{3}{*}{0.003467} & \multirow{3}{*}{0.005978} \\
\hline Grab-bike & 0.464650733 & & & \\
\hline Uber-bike & 0.136585911 & & & \\
\hline Direct Trips & Priority Vector & $\lambda \max$ & CI & $\mathbf{C R}$ \\
\hline Go-ride & 0.368936332 & \multirow{3}{*}{3.019325} & \multirow{3}{*}{0.009663} & \multirow{3}{*}{0.01666} \\
\hline Grab-bike & 0.4766732 & & & \\
\hline Uber-bike & 0.154390468 & & & \\
\hline Manueverability & Priority Vector & $\lambda \max$ & CI & $\mathbf{C R}$ \\
\hline Go-ride & 0.208211037 & \multirow{3}{*}{3.081301} & \multirow{3}{*}{0.04065} & \\
\hline Grab-bike & 0.702865435 & & & 0.070087 \\
\hline Uber-bike & 0.088923528 & & & \\
\hline
\end{tabular}


Based on the information of the table 17, 18, 19 and 20 above, we got the value of the consistency ratio. For criteria we got 0.079162 which is $<0,1$ or less than $10 \%$. For sub-criteria we got 0.06 for convenience; price 0.04 ; safety 0.04 ; speed 0.03 . Each sub-criteria was less than $10 \%$ which means that the findings are consistent. The consistency ratio of the alternatives appeared in the table 19 and 20 shows that the results are $<0.1$. It means that the results are consistent. It means that the research findings are consistent and that the researchers may continue the process of decision-making using AHP.

\section{Overall Priorities}

The next step is to determine overall priority for the alternatives. The calculation was done by first calculating weighted priorities of the alternatives with respect to the criteria and the sub criteria of each element, then summing the results to get the value of overall priorities of the alternatives.

Example calculation below can be found on determining the weighted priorities of the alternatives with respect to the convenience sub-criteria and criteria. To facilitate the calculation priority vector and the scalar are listed in the table 21, 22 and 23 below.

Table 21. Priorities matrix of the alternatives with respect to the convenience sub criteria $\left(w_{A C}\right)$

\begin{tabular}{|l|c|l|c|l|}
\hline CONVENIENCE & Pick \& Drop & $\begin{array}{l}\text { Easy to } \\
\text { Access }\end{array}$ & Privatization & Predictable \\
\hline Go-ride & 0.309120701 & 0.309592536 & 0.319093866 & 0.432526863 \\
\hline Grab-bike & 0.556602196 & 0.589395902 & 0.544962165 & 0.414648785 \\
\hline Uber-bike & 0.134277103 & 0.101011561 & 0.135943969 & 0.152824353 \\
\hline
\end{tabular}

Tabel 22. Priority vectors of the convenience sub criteria with respect to the convenience criteria $\left(w_{S c C}\right)$

\begin{tabular}{|l|c|}
\hline CONVENIENCE & Priority Vector \\
\hline Pick \& Drop & 0.356868 \\
\hline Easy to Acces & 0.407108 \\
\hline Privatization & 0.141828 \\
\hline Predictable & 0.094196 \\
\hline
\end{tabular}


Table 23. Priority scalar of the convenience criteria with respect to the goal

\begin{tabular}{|l|c|}
\hline CRITERIA & $\begin{array}{c}\text { Priority } \\
\text { Vector }\end{array}$ \\
\hline Convenience & 0.32241 \\
\hline
\end{tabular}

The calculation of the weighted priorities of alternatives with respect to the convenience subcriteria and criteria is as follows :

$$
\begin{gathered}
{\left[\begin{array}{c}
\text { Go - ride } \\
\text { Grab - bike } \\
\text { Uber - bike }
\end{array}\right]=\left\{\left(w_{A C}\right) \cdot\left(w_{S c C}\right)\right\} \cdot\left(w_{c}\right)} \\
{\left[\begin{array}{c}
\text { Go - ride } \\
\text { Grab - bike } \\
\text { Uber - bike }
\end{array}\right]=\left\{\left[\begin{array}{llll}
0.309120701 & 0.309592536 & 0.319093866 & 0.432526863 \\
0.556602196 & 0.589395902 & 0.544962165 & 0.414648785 \\
0.134277103 & 0.101011561 & 0.135943969 & 0.152824353
\end{array}\right] \cdot\left[\begin{array}{c}
0.356868 \\
0.407108 \\
0.141828 \\
0.094196
\end{array}\right]\right\} \cdot 0.32241} \\
{\left[\begin{array}{c}
\text { Go - ride } \\
\text { Grab - bike } \\
\text { Uber - bike }
\end{array}\right]=\left[\begin{array}{l}
0.103930356 \\
0.178916791 \\
0.039565853
\end{array}\right]}
\end{gathered}
$$

The calculation to get the other values of the weighted priorities associating criteria and the sub criteria are done in the same way and the result are listed in the table 24 below. Overall priority is obtained by the summing of the row in the table 24 below.

Table 24. Overall priorities for the alternatives with respect to the sub criterion and criterion

\begin{tabular}{|l|r|r|r|r|r|}
\hline alternatives & \multicolumn{1}{|c|}{ Convenience } & \multicolumn{1}{c|}{ Price } & \multicolumn{1}{c|}{ Safety } & \multicolumn{1}{c|}{ Speed } & \multicolumn{1}{c|}{ Overall Priority } \\
\hline Go-ride & 0.103930356 & 0.101095116 & 0.05259123 & 0.042916202 & $\mathbf{0 . 3 0 0 5 3 2 9 0 4}$ \\
\hline Grab-bike & 0.178916791 & 0.220367408 & 0.079181145 & 0.058729942 & $\mathbf{0 . 5 3 7 1 9 5 2 8 7}$ \\
\hline Uber-bike & 0.039565853 & 0.082408475 & 0.025396626 & 0.014901973 & $\mathbf{0 . 1 6 2 2 7 2 9 2 6}$ \\
\hline
\end{tabular}

The results of overall priority calculation, as mentioned in table 24 , showed that Grab bike obtained 54\% and can be considered as the highest priority, followed by Go-ride with $30 \%$ and Uber-bike with $16 \%$.

We can conclude that the dominant factor influencing people to use motorcycle taxi online service is price. The favorite motorcycle online service as perceived by the customers is Gabbike.

The complete results following convention and stating the local priorities can be seen in the table 25 below. 
Table 25. Overall priorities of the alternatives with respect to the sub-criteria and criteria

\begin{tabular}{|c|c|c|c|c|c|c|c|c|}
\hline \multirow{2}{*}{$\begin{array}{c}\text { Priority of } \\
\text { Criteria }\end{array}$} & \multicolumn{2}{|c|}{ Priority of Sub Criteria } & \multicolumn{3}{|c|}{ Priority of Alternatives } & \multicolumn{3}{|c|}{ Weighted Priority of Alernatives } \\
\hline & SubCriteria & priorities & Go-ride & Grab-bike & Uber-bike & Go-ride & Grab-bike & Uber-bike \\
\hline Convenience & Pick \& Drop & 0.356868 & 0.309120701 & 0.556602196 & 0.134277103 & \multirow{4}{*}{0.103930356} & \multirow{4}{*}{0.178916791} & \multirow{4}{*}{0.039565853} \\
\hline \multirow{3}{*}{0.322413} & Easy to Acces & 0.407108 & 0.309592536 & 0.589395902 & 0.101011561 & & & \\
\hline & Privatization & 0.141828 & 0.319093866 & 0.544962165 & 0.135943969 & & & \\
\hline & Predictable & 0.094196 & 0.432526863 & 0.414648785 & 0.152824353 & & & \\
\hline Price & Affordable & 0.650533 & 0.21109412 & 0.556069778 & 0.232836102 & \multirow{3}{*}{0.101095116} & \multirow{3}{*}{0.220367408} & \multirow{3}{*}{0.082408475} \\
\hline \multirow{2}{*}{0.403871} & Fixed & 0.232271 & 0.279721933 & 0.562211869 & 0.158066198 & & & \\
\hline & Flexible & 0.117196 & 0.409744188 & 0.454886294 & 0.135369518 & & & \\
\hline Safety & Traceable Route & 0.35353 & 0.288276668 & 0.539684521 & 0.172038811 & \multirow{5}{*}{0.05259123} & \multirow{5}{*}{0.079181145} & \multirow{5}{*}{0.025396626} \\
\hline \multirow{4}{*}{0.157169} & $\begin{array}{l}\text { Driver } \\
\text { Recognized }\end{array}$ & 0.2537008 & 0.348144669 & 0.49792467 & 0.153930661 & & & \\
\hline & Formal Office & 0.1583379 & 0.434390655 & 0.41074889 & 0.154860456 & & & \\
\hline & Valid License & 0.1201978 & 0.368936332 & 0.4766732 & 0.154390468 & & & \\
\hline & G.C. Vehicle & 0.1142328 & 0.273570297 & 0.563280372 & 0.163149331 & & & \\
\hline Speed & Realtime & 0.400495 & 0.413683584 & 0.459259484 & 0.127056932 & \multirow{4}{*}{0.042916202} & \multirow{4}{*}{0.058729942} & \multirow{4}{*}{0.014901973} \\
\hline \multirow{3}{*}{0.116548} & All Access & 0.266878 & 0.398763356 & 0.464650733 & 0.136585911 & & & \\
\hline & Direct Trips & 0.167189 & 0.368936332 & 0.4766732 & 0.154390468 & & & \\
\hline & Maneuverablity & 0.165439 & 0.208211037 & 0.702865435 & 0.088923528 & & & \\
\hline \multicolumn{6}{|c|}{ Overall Priorities of Alternatives } & 0.3005329 & 0.53719529 & 0.162272926 \\
\hline
\end{tabular}

\section{Conclusion}

The study shows that price is considered at the dominant factor for a customer looking for motorcycle service online followed convenience, safety and speed. It means that most customers are price sensitive. The element of price which is dominant for customers was affordability. The customers considered that affordable price is the most important factor for them to use online motorcycle service. Out of the three online motorcycles taxi service providers that operate in Manado, the Grab-bike is preffered by customers. The researchers also found out in the field that Grab-bike motorcycle taxi online services always provide discount prices for customers. The study is considered valid since the consistency ratio of each criteria, sub-criteria and alternative is less than 0.1 or $(10 \%)$. 


\section{References}

Banuelas, R., Anthony, J. (2004). Modified Analytical Hierarchy Process to incorporate uncertainty and managerial aspects. Int. J. Prod. Res, Vol 42, No.18, pp. 3851-3872

Karema, F.M. (2013). The Role of Commercial Motorcycles in Rural Economy. A Case Study of Laikipia East Sub-County Kenya. University of Nairobi. \{Online http://geography.uonbi.ac.ke/sites/default/files/chss/arts/geography/2015\%20Abstract\%2 OKAREMA\%20FREDRICK\%20MWANGI.pdf (accessed 12 January 2018).

Keong, W. E. Y. (2015). Factors Influencing Malaysian Taxi Drivers Behavioral Intention to Adapt Mobile Taxi Application. International Journal of Economics, Commerce and Management, Vol III, Issue 11, pp. 139-156

Kumar, A. (2011). Understanding the Emerging Role of Motorcycles in African Cities. \{Online) https://www.ssatp.org/sites/ssatp/files/pdfs/Topics/urban/DP13-Role Motorcycles\%5B1\%5D.pdf (accessed 10 January 2018).

Liu, Z.Y. (2014). An Analysis of Technology Acceptance Model. Exploring user acceptance and intension of taxi-hailing app in Shanghai. University of Gothenburg. \{Online https://gupea.ub.gu.se/bitstream/2077/38592/1/gupea_2077_38592_1.pdf (accessed 20 December 2017).

Qian, J. (2015). Disciplined Mobility and Migrant Subalternity: Scetching the Politics of Motorcycle Taxis in Guangzhou. Urban Studies, 52 (15), pp. 2922 - 2947

Raco, J.R. and Tanod, R.H.M. (2014). 'The phenomenological method in entrepreneurship, Int. J. Entrepreneurship and Small Business, Vol. 22, No.3, pp. 276-28

Shah, G.U.D., Bhatti, M.N., Iftikhar, M., Qureshi M.I., Zaman, Kh. (2013). Implementation of Technology Acceptance Model in E-learning Environment in Rural and Urban areas of Pakistan. World Applied Sciences Journal, 27 (11), pp. 1495-1507.

Sinuany-Stern, Z., Israeli, Y., Bar-Eli, M. (2006). Application of analytical hierarchy process for the evaluation of basketball teams, Int. J. Sport Management and Marketing, Vol 1, No.3, pp. 193-207.

Sodikin, A (2017). Kompas daily news, 14 December 2017, p. 6

Zhang, G., Ma, L.,Zhang, X. (2017). In: Wahan International Conference on e-Business. Association for Information Systems, pp 231-240. 